\title{
Brigada de Primeros Auxilios Psicológicos: Experiencia en el Simulacro NeONATAL de EXPANSIÓN HOSPITALARIA DEL INPER
}

\author{
Diana Pimentel Nieto y Mauricio Ramos Cruz \\ Instituto Nacional de Perinatología Isidro Espinosa de los Reyes \\ México
}

\begin{abstract}
RESUMEN
El Instituto Nacional de Perinatología "Isidro Espinosa de los Reyes" es reconocido por su trabajo multidisciplinario incorporando diversas profesiones, entre ellas la Psicología, dirigiendo sus objetivos hacia la optimización de los recursos psíquicos de los pacientes y/o personal, donde en una situación de desastre no tendría que ser la excepción, creándose la brigada de Primeros Auxilios Psicológicos.Para exponer el trabajo desarrollado por la brigada y la importancia del quehacer psicológico, se explicará el adiestramiento efectuado conforme al protocolo establecido el día jueves 9 de agosto de 2018 a las 11 horas en el primer simulacro neonatal con expansión hospitalaria, con la hipótesis de un sismo de magnitud 7.7, teniendo un total de 151 participantes pertenecientes a las 10 brigadas del Instituto y 25 observadores externos (Flores, 2018), suceso durante el cual se consolidaron los objetivos de la brigada de primeros auxilios psicológicos, dando como resultado la mitigación del malestar emocional de las mujeres en estado de crisis mediante la aplicación de recursos teórico-metodológicos de intervención en crisis, además de hacer un análisis riguroso del suceso para la creación de un informe que sirvió de retroalimentación y promover la importancia al cuidado de la salud mental.
\end{abstract}

Palabras Clave:

psicología, desastre, salud mental, brigadas, crisis

\section{Psychological First Aid BRIgade: EXPERIENCE IN THE INPeR'S Neonatal Simulation of Hospital Expansion}

\begin{abstract}
The National Institute of Perinatology "Isidro Espinosa de los Reyes", is recognized for its multidisciplinary work, incorporating various professions, including Psychology, directing its objectives towards optimizing the psychic resources of patients and/or staff; where in a disaster situation it should not be the exception, creating the Psychological First Aid brigade. To explain the work carried out by the brigade and the importance of psychological work, the training carried out in accordance with the established protocol will be explained on Thursday, August 9, 2018 at 11:00 am, in the first neonatal drill with hospital expansion, under the hypothesis of an earthquake with a magnitude of 7.7, having a total of 151 participants, belonging to the 10 Brigades of the Institute and 25 external observers (Flores, 2018), an event during which the objectives of the first aid brigade were consolidated psychological, resulting in the mitigation of emotional distress of women in crisis by applying theoretical-methodological resources of crisis intervention, in addition to conducting a rigorous analysis of the event for the creation of a report that served as feedback and finally promote the importance of mental health care.
\end{abstract}

Keywords:

psychology, disaster, mental health, brigades, crisis

Bitácora del Artículo:

| Recibido: 21 de enero de 2019 | Aceptado: 8 de Junio de 2020 | Publicado en línea: Julio-Diciembre de 2020 | 


\title{
Brigada de Primeros Auxilios Psicológicos: EXPeriencia en el Simulacro NeONATAL de EXPANSIÓN HospitAlaria dEL INPER
}

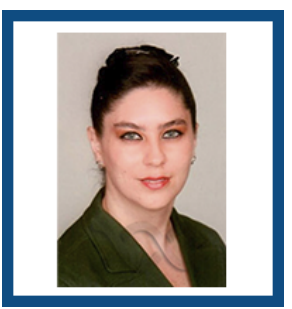

Diana Pimentel Nieto

INPER

Correo: dpimenteln@yahoo.com

Maestra en Psicología General Experimental con Énfasis en Salud, egresada de la Facultad de Psicología de la Universidad Nacional Autónoma de México. Adscrita al Departamento de Consulta Externa en el Instituto Nacional de Perinatología como investigadora en Ciencias Médicas "B". Docente del Centro Universitario ELEIA en la práctica privada.

\author{
Diana Pimentel Nieto y Mauricio Ramos Cruz \\ Instituto Nacional de Perinatología Isidro Espinosa de los Reyes \\ México
}

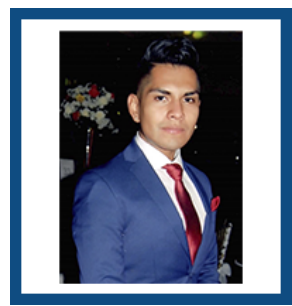

Mauricio Ramos Cruz

INPER

Correo:psic.mau.puma@hotmail.com

Licenciado en Psicología de la Universidad Nacional Autónoma de México. Hizo su servicio social en el Instituto Nacional de Perinatología Isidro Espinosa de los Reyes, donde funge como asistente de investigación en el programa de "Vigilancia Epidemiológica en Salud Mental" en el servicio de Psicología Consulta Externa. Técnico en banco de sangre, desarrollando servicio social en el Hospital General de México "Dr. Eduardo Liceaga”.

\section{CONTRIBUCIÓN DE LOS AUTORES}

Diana Pimentel Nieto trabajó en la actuación junto con los brigadistas para el apoyo en la estabilización emocional hizo análisis de los datos obtenidos mediante la observación participante para recomendaciones a la intervención y al actuar del factor humano, a su vez efectuó la revisión y supervisión del manuscrito. | Mauricio Ramos Cruz coordinó y desarrolló las actividades del proyecto de la brigada de los primeros auxilios psicológicos, recabó y analizó los datos obtenidos de la actuación de los brigadistas e integró el documento recabando la información para la presentación del trabajo.

\section{AGRADECIMIENTOS}

Gracias al Instituto Nacional de Perinatología "Isidro Espinosa de los Reyes" en conjunto con el Director General, el Director de Administración y Finanzas y la Jefa del Departamento de Capacitación y Desarrollo Personal, quienes nos han permitido colaborar para el beneficio integral de la población atendida y el personal del Instituto. De igual forma agradecemos y otorgamos un digno reconocimiento a la Unidad Interna de Protección Civil ante su labor, la cual es una tarea ardua y exigente, que sin su apoyo este trabajo no podría haberse consolidado.

\section{DATOS DE Filiación DE LOS Autores}

Instituto Nacional de Perinatología Isidro Espinosa de los Reyes

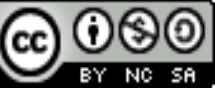

Copyright: (c) 2020 Pimentel-Nieto, D. \& Ramos-Cruz, M.

Este es un artículo de acceso abierto distribuido bajo los términos de la licencia Creative Commons Reconocimiento-NoComercial 4.0 Internacional, por lo que su contenido gráfico y escrito se puede compartir, copiar y redistribuir total o parcialmente sin necesidad de permiso expreso de sus autores con la única condición de que no se puede usar con fines directamente comerciales y los términos legales de cualquier trabajo derivado deben ser los mismos que se expresan en la presente declaración. La única condición es que se cite la fuente con referencia a la Revista Digital Internacional de Psicología y Ciencia Social y a sus autores. 


\section{TABLA DE CONTENIDO}

\section{INTRODUCCIÓN}

Alcance de la psicología en la hospitalización, 418

La incorporación de la psicología en situaciones de emergencia, 419

MÉTODO

Identificación del caso, 419

Análisis del motivo de la intervención, 420

Antecedentes del problema, 420

Descripción y análisis del problema, 420

\section{PLAN DE INTERVENCIÓN}

Descripción del plan intervención, 422

Establecimiento de los objetivos y las metas del plan de

intervención, 422

Técnicas de evaluación del plan de intervención, 422

EFICACIA DEL PLAN DE INTERVENCIÓN

Presentación de resultados, 423

Análisis de los cambios que produjo el plan de intervención, 423

Significancia clínica de los cambios, 423

Relevancia social del cambio, 423

\section{Pronóstico}

Resultados en la fase de seguimiento, 424

\section{REFERENCIAS}

\section{REFERNCIAS}




\section{INTRODUCCIÓN}

M éxico es un país que en los últimos años ha especificado sus prioridades en salud, porque de ello depende en gran medida la posibilidad de ofrecer una respuesta a las demandas y necesidades de la población, así como de las políticas estratégicas nacionales que permitan mejores condiciones de vida para el país. Por ello se creó el Instituto Nacional de Perinatología Isidro Espinosa de los Reyes, el cual es una institución de tercer nivel en atención médica. Hoy se caracteriza por ser un instituto de los más grandes y por presentar diversos reconocimientos a su actuar en beneficio de la población gineco-obstétrica; sin embargo, podemos mencionar que este avance ha requerido su proceso a lo largo del tiempo.

El antecedente más antiguo a la atención a dicha población se remonta a 1921, cuando se edifica la Casa del periodista, de carácter privado para el apoyo a la maternidad, y que en 1929 pasó a ser una beneficencia pública; en 1976 se expropió el predio ocupado por la Maternidad Isidro Espinosa de los Reyes, que es dependiente de la Secretaría de Salubridad y Asistencia, para formar parte de la Institución Mexicana de Asistencia a la Niñez, donde tiempo después, al considerar que dichas instituciones compartían objetivos y programas en común, se fusionaron por decreto del 10 de enero de 1977, constituyendo el Sistema Nacional para el Desarrollo Integral de la Familia (DIF) y el Instituto Nacional de Perinatología Isidro Espinosa de los Reyes (INPer), el cual se inauguró el 8 de diciembre de 1977 con la dirección del doctor Eduardo Jurado García (INPer, 2017).

Comenzando su recorrido desde tiempo atrás y como en cualquier institución, tiene objetivos específicos, su misión y su visión (INPer, 2017).

Su misión se enfoca a la generación de nuevo conocimiento mediante la innovación e investigación de calidad que impacta en la población, formación del talento humano de alta especialidad y de atención médica de gran complejidad en el ámbito de la salud reproductiva y perinatal, para erigirse como modelo referente de salud a nivel nacional, marcando directrices para contribuir en la alineación de políticas nacionales de salud para el bienestar de la sociedad.

Su visión busca ser una institución líder que determina las pautas a seguir en salud reproductiva y perinatal con estándares bioéticos y de calidad, aplicando valores de huma- nismo, calidez, honestidad, responsabilidad, respeto y resiliencia en la investigación, enseñanza y atención médica.

Sus objetivos constan en realizar estudios e investigaciones clínicas, epidemiológicas, experimentales de desarrollo tecnológico y básicas en las áreas biomédicas y sociomédicas en el campo de su especialidad para la comprensión, prevención, diagnóstico, tratamiento de enfermedades y rehabilitación; publicar los resultados de las investigaciones y trabajos que se desarrollen, promover y hacer reuniones de intercambio científico, de carácter nacional e internacional, formar recursos humanos en sus áreas de especialización, formular y aplicar programas de estudio y cursos de capacitación, enseñanza, especialización y actualización del personal profesional, otorgar constancias, diplomas, reconocimientos y certificados de estudios, grados y títulos, ofrecer servicios de salud en aspectos preventivos, médicos, quirúrgicos y de rehabilitación, proporcionar consulta externa, atención hospitalaria y servicios de urgencias a la población que requiera atención médica en sus áreas de especialización, asesorar y formular opiniones a la Secretaría cuando sea requerido, actuar como órgano de consulta, técnica y normativa de las dependencias y entidades de la administración pública, asesorar a los centros especializados de investigación, enseñanza o atención médica, promover acciones para la protección de la salud, coadyudar con la Secretaria a la actualización de los datos respecto a la situación sanitaria, y desarrollar las demás actividades que le correspondan conforme a la ley y otras disposiciones aplicables.

Aunado a ello se reconoce su actuar multidisciplinario, colaborando en la formación de recursos humanos especializados en todas las disciplinas vinculadas en el área de la salud, dentro de éstos la Psicología, donde los objetivos del trabajo psicológico en primera instancia se enfocan en la optimización de los recursos psíquicos de los pacientes frente a la salud sexual y reproductiva, buscando una formación biopsicosocial, apoyando el mejoramiento asistencial de los pacientes, así como siendo un facilitador de la asistencia psicoterapéutica para el trabajo multidisciplinario, respondiendo a las necesidades institucionales y poblacionales ante su dolencia o evento biológico que se cursa, buscando lograr un bienestar de salud integral (Espíndola et al., 2006).

\section{Alcance de la psicología en la hospitalización}

El INPer se caracteriza por ser un hospital de tercer nivel que busca el apoyo a pacientes gineco-obstétricas que acuden por diferentes padecimientos de alto riesgo, ya sea por embarazo y/o enfermedad, teniendo como consecuencia en la mayoría de las pacientes un tipo de malestar psicoló- 
gico no patológico secundario al padecimiento médico y/o hospitalización, mientras que la minoría de la población llega a presentar un padecimiento psicopatológico exacerbado por la circunstancia vivida (Espíndola et al., 2006).

Para ello se reconoce la inserción del trabajo psicoterapéutico en instituciones hospitalarias no psiquiátricas donde el experto en salud mental ofrecerá el apoyo adecuado para su evaluación y la implementación de estrategias con técnicas específicas, las cuales facilitarán la solución al conflicto presentado con la realidad o de manera intrapsíquica por las complicaciones presentadas en un proceso de atención gineco-obstétrico, donde un punto clave ha sido la promoción de la inserción de la psicología en dichos ámbitos, obteniendo un abordaje de manera integral al paciente, mejorando su adherencia terapéutica y conciencia de salud o de enfermedad (Morales-Carmona y Díaz-Franco, 2000).

\section{La incorporación de la psicología situaciones de emergencia}

A lo largo del tiempo hemos estado expuestos a diversas situaciones de emergencia a las que hemos tenido que dar respuesta sobre todo desde el aspecto físico y económico; sin embargo, la intervención puede ir más allá de estos factores. La Real Academia Española (2020) define el concepto "emergencia" como cualquier situación de peligro o desastre que requerirá una acción inmediata.

Se conoce que los desastres pueden llegar a ser inminentes porque pueden surgir mediante diversos eventos, ya sean basados en la naturaleza, en la violencia social, la degradación ambiental y la privación económica, lo que generará un impacto potencial para la población afectada (Tamariz, 2018) y que requerirá atención especializada en diversas áreas.

La Organización Mundial de la Salud (OMS, 2020), indica que durante una situación de emergencia operan factores sanitarios y sociales específicos, de manera incluyente o excluyente, refiriéndose, a manera de ejemplo, a los siguientes factores.

- Salud física.

- Salud bucodental y el estado de la dentadura.

- Salud mental.

- Capacidad funcional y discapacidades.

- Modos de vida.

- Nutrición.

- Relaciones familiares y sociales.

- Situación económica.

- Cuestiones de género.

Abocándonos hacia el factor de la salud mental, el cual ha tenido relevancia en los últimos años, ha proporcionado respuestas para la intervención en escenarios de emergencias, incorporando al quehacer psicoterapéutico para el apoyo y optimización de los recursos psíquicos de las personas afectadas, donde a manera de respuesta inmediata surgen los primeros auxilios psicológicos.

Rabelo (2010) menciona que los primeros auxilios psicológicos se caracterizan por ser intervenciones inmediatas y de corta duración, las cuales estarán dirigidas hacia las personas afectadas por un incidente crítico, buscando proporcionar apoyo mediante la expresión de los sentimientos, la facilitación de búsqueda de soluciones y apoyo social, la recuperación en su funcionamiento previo al incidente, la reducción del riesgo de muerte, la prevención de consecuencias negativas hacia su salud mental, y ser un facilitador de enlace con los recursos de ayuda.

Martínez y Pery (2013), psicólogos de las Fuerzas Armadas de España, refieren que los primeros auxilios psicológicos se utilizan como una intervención de tipo psicosocial que se puede proporcionar, por personal capacitado en el área, a cualquier persona implicada en una crisis, con el propósito de ofrecer apoyo emocional, donde los especialistas (médicos, psicólogos, enfermeros y trabajadores sociales) tienen una función fundamental.

Rotger (1999), psicólogo del Cuerpo de Bomberos de Palma de Mallorca, refiere que es necesario el apoyo de especialistas en el área de la salud mental porque ayudar a una víctima va más allá de alejarla del peligro, momento en el cual su situación psíquica se encuentra temporalmente desordenada y la incompetencia en estas situaciones tendría consecuencias graves para el afectado, porque en la manera que la persona afectada enfrente un percance en su vida será determinante para su futuro.

Amenero y Huarcaya (2019) indican que los problemas psicosociales y los desastres pueden ser un factor de incidencia en los trastornos mentales ante los horrores vividos en las emergencias, las pérdidas experimentadas, incertidumbres y estresores, alterando el significado y el propósito de vida; entre los principales trastornos psiquiátricos secundarios al desastre se identifican el trastorno de estrés postraumático, trastorno depresivo y trastorno por consumo de sustancias.

\section{Método}

\section{Identificación del caso}

México se concibe como una población expuesta a diversos eventos de desastre, lo que genera un alto grado de vulnerabilidad ya sea física y/o social. Por ello es de suma relevancia contar con organizaciones especializadas que se encarguen de la atención a las necesidades específicas del suceso, generando acciones, normas y 
programas destinados para prevenir y controlar los efectos causados (Centro Nacional de Prevención de Desastres, 2018), donde no se puede dejar de lado el interés hacia la salud mental.

Por lo indicado se considera de relevancia poner atención al modo de intervención que se tendría en situaciones de desastres desde una perspectiva multidisciplinaria, cubriendo todos los aspectos para una atención integral del factor humano desde un aspecto biopsicosocial, donde en el Instituto Nacional de Perinatología adquiere gran relevancia al pensar que su población se considera vulnerable ante los padecimientos médicos presentados, y que ello podría ser un factor de riesgo para los estados de crisis en situaciones de desastre.

\section{Análisis del motivo de la intervención}

Es sabido que los efectos o repercusiones que se presentan posterior a un desastre son diversos; entre ellos cabe destacar las repercusiones intrapsíquicas generadas por el impacto al evento, donde se puede presentar una desorganización temporal en las personas ocasionando estados de crisis al sobrepasar sus recursos para afrontar las adversidades y, de no ser atendidas, pueden llegar a presentar alteraciones significativas en su funcionamiento cotidiano (CENAPRED, 2018).

Por ello es necesario un organismo encargado para la atención especializada hacia la salud mental tanto para la población afectada como para los agentes reguladores de las acciones, donde el psicólogo desarrolla una función primordial, por lo que se expondrá la experiencia obtenida de manera institucional por parte de la brigada de primeros auxilios psicológicos, ejemplificando la participación del trabajo psicológico durante el primer Simulacro Neonatal de Expansión Hospitalaria en el INPer.

\section{Antecedentes del problema}

El origen de la brigada de primeros auxilios psicológicos dentro del instituto se remonta a la participación del personal con la asistencia a cursos y capacitaciones para el apoyo a las acciones promovidas por el grupo de Protección Civil; sin embargo, en 2013 se hizo la designación oficial para la creación de la brigada con la dirección de la maestra Diana Pimentel Nieto como jefa de dicha brigada.

El acontecimiento fue consolidado gracias a la participación y apoyo por parte de los directivos, el Director General, la Jefa del departamento de Capacitación, Calidad y Desarrollo de Personal, el grupo de Protección Civil, los jefes de brigadas y los brigadistas, estableciendo la organización para salvaguardar la integridad en salud mental de la población del Instituto en situaciones de desastre; sin embargo conocemos que la voluntad de ayudar no es suficiente, por lo que el brigadista de Primeros Auxilios Psicológicos deberá estar capacitado y especializado para atender situaciones de crisis y emergencias, evitando un efecto contraproducente de la intervención.

El brigadista de Primeros Auxilios Psicológicos deberá tener un perfil activo y empático, manteniendo un rol de acompañante para la contención emocional y siendo un promotor de catarsis. Para ello se implementa la formación continua del personal que participa en estas acciones enfocadas a salvaguardar el equilibrio emocional, que se desarrolla mediante prácticas, cursos, capacitaciones e informes, entre otras actividades que optimicen el rendimiento del personal para una actuación propicia, donde la participación en un simulacro ofrece la oportunidad para la práctica integral del conocimiento adquirido. Además se ofrecen capacitaciones para el conocimiento básico de estrategias de actuación de las demás brigadas, fortaleciendo habilidades para la resolución del incidente crítico, por lo cual el departamento de Protección Civil del Instituto ha otorgado capacitaciones en los siguientes temas: 1) uso y manejo de extintores y códigos de emergencias; 2) criterios de evacuación; 3) manejo de sustancias químicas peligrosas; 4) intervenciones de enfermería en caso de desastre; 5) formación e integración de brigadas; 6) evacuación aeromédica; 7) primeros auxilios psicológicos; 8) programa de atención a la población; 9) comunicación en emergencias; 10) sistema de comando de incidentes, y 11) fundamentos de la atención pre hospitalaria, entre otras.

\section{Descripción y análisis del problema}

El día jueves 9 de agosto de 2018, a las 11 horas se efectuó el primer simulacro neonatal con expansión hospitalaria en el Instituto Nacional de Perinatología "Isidro Espinosa de los Reyes", con la hipótesis de un sismo de magnitud 7.7, con 151 participantes (tabla 1 y figura 1), pertenecientes a las 10 brigadas del Instituto (Brigada de Evacuación Hospitalaria, Brigada de Soporte Básico de Vida, Brigada de Prevención y Combate de Incendios, Brigada de Comunicación, Brigada de Seguridad, Brigada de Fluidos y Energéticos, Brigada de Búsqueda y Salvamento, Brigada de Primeros Auxilios Psicológicos, Brigada de Riesgos Químicos, Brigada Multifuncional del Turno Vespertino, Nocturno y Fin de Semana) y 25 observadores externos (tabla 2 y figura 2) de diversas instituciones (Instituto Nacional de Pediatría, Instituto Nacional de Rehabilitación, Instituto Nacional de Enfermedades Respiratorias, Hospital Infantil de México Federico Gómez, Grupo Cóndores SSP, Erum SSP, Tránsito SSP, Protección Civil SSP, Bomberos y Protección Civil Miguel Hidalgo) (Flores, 2018). 
Tabla 1.

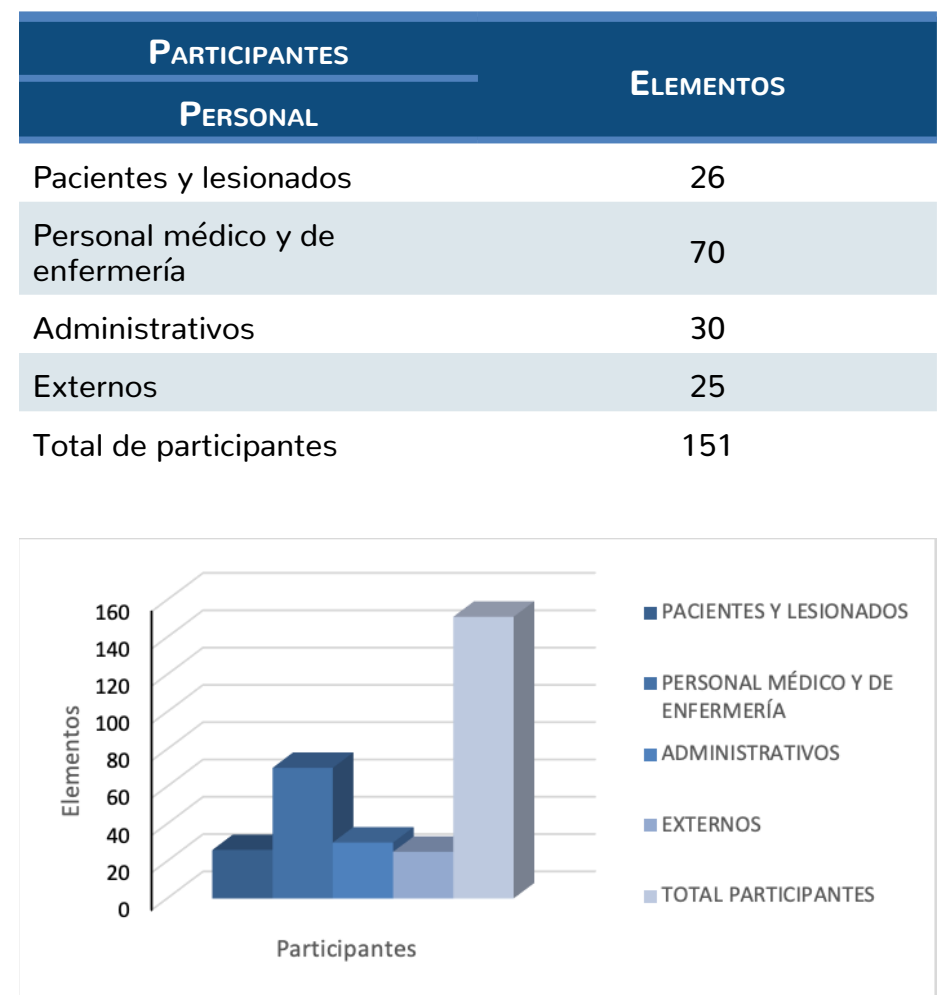

Figura 1.

Tabla 2.

\begin{tabular}{|c|c|}
\hline Observadoores externos & \multirow{2}{*}{ ELementos } \\
\hline INSTITUCIÓN & \\
\hline Instituto Nacional de Pediatría & 2 \\
\hline $\begin{array}{l}\text { Instituto Nacional de } \\
\text { Rehabilitación }\end{array}$ & 4 \\
\hline $\begin{array}{l}\text { Instituto Nacional de } \\
\text { Enfermedades Respiratorias }\end{array}$ & 3 \\
\hline $\begin{array}{l}\text { Hospital Infantil de México } \\
\text { Federico Gómez }\end{array}$ & 2 \\
\hline Grupo Cóndores SSP & 2 \\
\hline Erum SSP & 1 \\
\hline Tránsito SSP & 4 \\
\hline Protección Civil SSP & 3 \\
\hline Bomberos & 1 \\
\hline $\begin{array}{l}\text { Protección Civil Miguel } \\
\text { Hidalgo }\end{array}$ & 5 \\
\hline Total & 25 \\
\hline
\end{tabular}

Conforme al protocolo establecido se comenzó por la ejecución del repliegue en las distintas áreas involu- cradas, la revisión del inmueble que indicó un daño estructural, lo que dio inicio a la evacuación de las áreas neonatales para continuar con la expansión hospitalaria y ofrecer las atenciones necesarias en los puntos estratégicos de cada brigada, como fue indicando en los cursos otorgados por el Centro Regulador de Emergencias Industriales Javier de la Madrid Torices (2018) y Bc Business Consultants Management S.C. (2018) con el tema "Comando de incidentes", entre otras conferencias como la impartida por Segundo (2018) con la temática "Efectos de las emergencias y desastres en los servicios de salud".

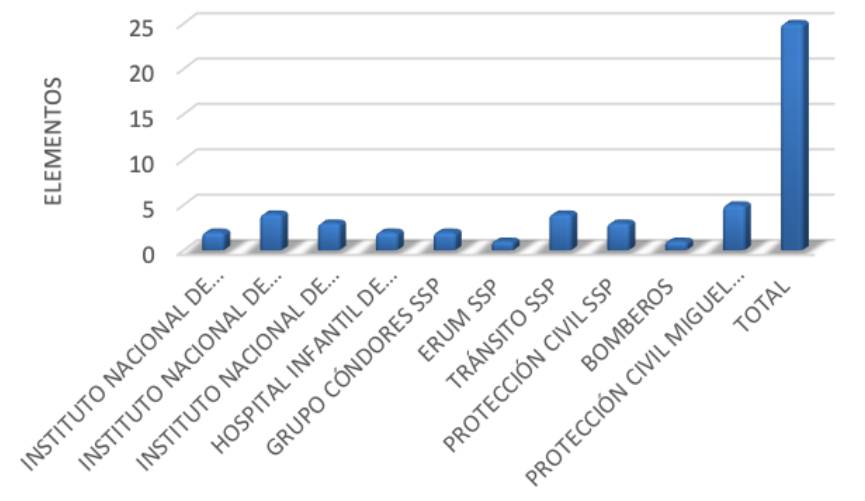

Figura 2.

Al término de la actividad se logró la atención a 26 pacientes, donde dos de ellos eran mujeres en aparente estado de crisis (tabla 3 y figura 3); otros tres necesitaron ser trasladados en dos ambulancias terrestres y una área al Hospital de Traumatología Lomas Verdes, Hospital Balbuena y al Instituto Nacional de Pediatría por condiciones fisiológicas (tabla 4).

Tabla 3.

\begin{tabular}{|c|c|}
\hline Pacientes & \multirow{2}{*}{ Elementos } \\
\hline Desalojados & \\
\hline Neonatos rojos & 3 \\
\hline Neonatos amarillos & 8 \\
\hline Neonatos verdes & 10 \\
\hline Adultos lesionados & 3 \\
\hline $\begin{array}{l}\text { Adultos psicológicamente en } \\
\text { crisis }\end{array}$ & 2 \\
\hline Erum SSP & 1 \\
\hline Tránsito SSP & 4 \\
\hline Protección Civil SSP & 3 \\
\hline Bomberos & 1 \\
\hline
\end{tabular}




\begin{tabular}{lcc}
\multicolumn{1}{c}{ Pacientes } & \\
\hline \multicolumn{1}{c}{ Desalojados } & Elementos \\
\hline $\begin{array}{l}\text { Protección Civil Miguel } \\
\text { Hidalgo }\end{array}$ & 5 \\
\hline & Total & 26 \\
\hline
\end{tabular}

Tabla 4.

\begin{tabular}{|lll}
\hline \multicolumn{1}{|c}{ Traslados } & \multicolumn{1}{c}{ Transporte } & \multicolumn{1}{c}{ ReCEPTOR } \\
\hline 1 Femenina & $\begin{array}{l}\text { Ambulancia } \\
\text { terrestre }\end{array}$ & $\begin{array}{l}\text { Traumatología } \\
\text { Lomas Verdes }\end{array}$ \\
\hline 1 Femenina & Ambulancia aérea & Hospital Balbuena \\
\hline $\begin{array}{l}\text { 1 Neonato } \\
\text { masculino } \\
\text { (gastrosquisis) }\end{array}$ & $\begin{array}{l}\text { Ambulancia } \\
\text { terrestre }\end{array}$ & $\begin{array}{l}\text { Instituto Nacional } \\
\text { de Pediatría }\end{array}$ \\
\hline
\end{tabular}

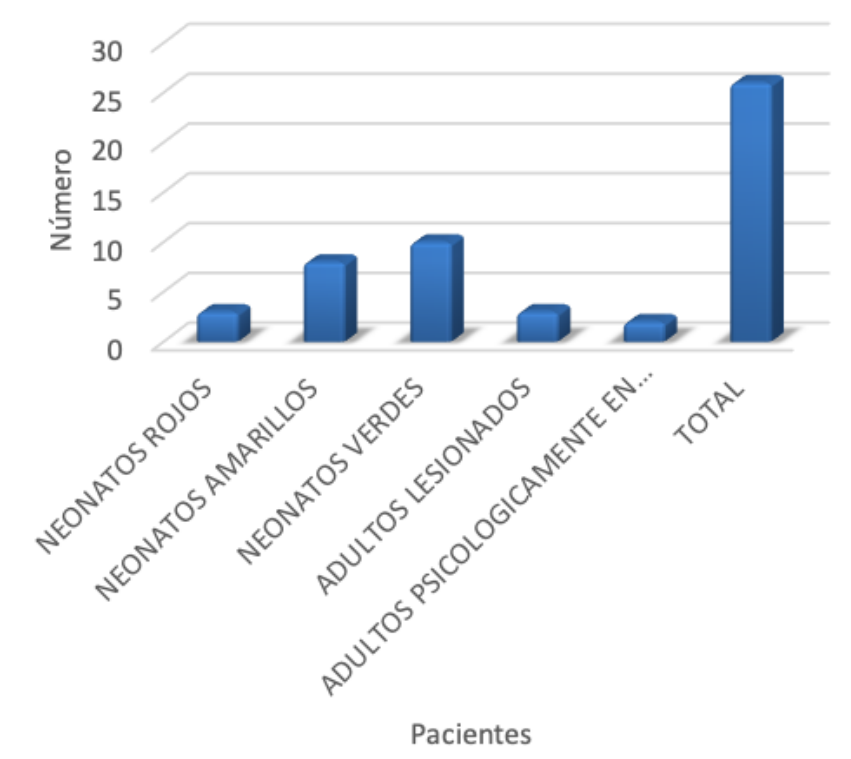

Figura 4.

\section{Plan de intervención}

\section{Descripción del plan intervención}

Hoy la brigada de Primeros Auxilios Psicológicos trabaja en dos direcciones. La primera se encarga de la atención hacia los pacientes y personal de la institución involucrados en algún incidente crítico, donde algunos se perciben incapacitados para afrontar el estado de tensión, lo que puede generar una crisis de ansiedad; dicha atención es proporcionada de manera inmediata, usando técnicas destinadas para reducir la ansiedad y fomen- tar la adaptación mediante estrategias de afrontamiento a corto, mediano y largo plazos. Es necesario indicar que la experiencia institucional ha enseñado que pese al conocimiento de que la actividad corresponde a un simulacro, es factible que haya personas que presenten ansiedad ante la práctica, por lo cual los brigadistas deberán estar altamente capacitados para enfrentar cualquier situación adversa que ponga en peligro la integridad psíquica de la persona durante el evento crítico.

La segunda línea de acción trabaja de manera simultánea con la primera, enfocándose en el análisis riguroso y exhaustivo del factor humano en las diversas brigadas de Protección Civil, observando el comportamiento y la participación con el objetivo de proponer estrategias efectivas para la actuación grupal adecuada y la resolución eficaz ante la problemática de un suceso real o en un simulacro.

\section{Establecimiento de los objetivos y las metas del plan de intervención}

- Buscar la estabilización del factor humano, quienes cursan estados de crisis.

- Analizar la actuación del factor humano, mediante una observación presente, en un evento real o simulado.

- Buscar la eficacia y eficiencia en el actuar de los brigadistas.

- Detectar perfiles de liderazgo en los brigadistas.

- Poner en práctica los conocimientos teórico-metodológicos para la resolución de la problemática.

- Concientizar al personal institucional y a la población atendida acerca de la importancia de la prevención en situaciones de desastre.

- Concientizar al personal institucional y a la población atendida respecto a los protocolos de actuación en situaciones de desastre.

- Promover la importancia de la salud mental.

\section{Técnicas de evaluación del plan de intervención}

- Seguimiento del protocolo de actuación ante una situación de desastre.

- Utilización de códigos de comunicación.

- Trabajo multidisciplinario.

- Contención emocional.

- Intervención en crisis.

- Técnicas de respiración.

- Facilitador comunicacional entre brigadas.

- Análisis observacional de manera presencial.

- Creación de informes para la retroalimentación institucional. 


\section{Eficacia del plan de intervención}

\section{Aplicación del plan de intervención}

En este simulacro se atendió a dos mujeres con una supuesta alteración emocional de tipo ansiosa reactiva al incidente crítico por el desconocimiento de la localización y el bienestar de sus bebés, por lo cual se practicaron acciones de intervención en crisis, contención emocional, acompañamiento, técnicas de respiración y se facilitó la intercomunicación entre las brigadas para mitigar la ansiedad de las pacientes. A la par se efectuó la observación y análisis de la participación del personal en la simulación para generar recomendaciones.

\section{Presentación de resultados}

Al término de la simulación se lograron los dos objetivos principales establecidos por la brigada de Primeros Auxilios Psicológicos: 1) la práctica exitosa de la estabilización emocional de las mujeres, mitigando el malestar emocional mediante el uso de las técnicas conocidas por los brigadistas, y 2) se hizo el análisis del simulacro, generando un informe que sirvió de retroalimentación para el personal del Instituto y que ha sido utilizado como un proceso de mejora en la planeación estratégica para la actuación ante eventos semejantes.

\section{Análisis de los cambios que produjo el plan de intervención}

Como efecto al simulacro se percibe la motivación del personal para la toma de conciencia preventiva de los incidentes críticos, se observaron perfiles de liderazgo, actuación según los protocolos establecidos por Protección Civil, el uso apropiado de los materiales de apoyo y la creatividad ante situaciones inesperadas.

\section{Significancia clínica de los cambios}

Podemos indicar que los procesos de actuación implementados por la brigada de Primeros Auxilios Psicológicos, en conjunto con el área de Protección Civil, ha generado un avance e impacto en la percepción a la prevención de riesgos y la importancia de la salud mental en la población del Instituto, reconociendo la importancia de la inclusión del trabajo psicoterapéutico en diversos ámbitos, entre ellos en las situaciones de desastre, donde el principal objetivo de las brigadas se enfoca a salvaguardar la vida física y psíquica del factor humano. Aunado a esto se reconoce el efecto de las capacitaciones brindadas y simulacros efectuados, pudiendo contar con personal capacitado en diversas áreas de actuación, fo- mentando el trabajo multidisciplinario y reconociendo la efectividad de la práctica previa a una emergencia.

Asimismo recomendamos continuar con la práctica de la comunicación efectiva entre las brigadas, clarificar los roles a desempeñar en los distintos grupos y el conocimiento del protocolo de actuación a nivel Institucional.

\section{Relevancia social del cambio}

Se infiere que pese al estigma social que se tiene hacia el trabajo psicoterapéutico, se ha logrado incorporar y reconocer la labor de la psicología en diversos ámbitos, siendo ésta un gran ejemplo de su reconocimiento al actuar, contando con personal capacitado, promoviendo y salvaguardando la importancia que se tiene hacia la salud mental, donde en concordancia con Rotger (1999), Amenero y Huarcaya (2019) se conoce que una mala actuación tendría graves repercusiones en el estado psíquico de las personas afectadas, por lo cual la atención a la salud mental no podemos verla como ajena al individuo, reconociendo sólo a la salud física.

Ante ello, el Instituto Nacional de Perinatología ha sido elogiado por su trabajo de manera multidisciplinaria, donde la psicología se ha incorporado en primera instancia para la atención a las pacientes gineco-obstétricas en un ámbito hospitalario no psiquiátrico, y donde hoy se ha generado mayor incorporación en diversas áreas para el quehacer del psicólogo, pudiendo referir a la creación de la brigada Primeros Auxilios Psicológicos, la cual, al ser un área que aún se encuentra en líneas de desarrollo, ha logrado obtener diversos resultados positivos para la atención de los ciudadanos, reconociendo la importancia de la atención psicológica. Aunado a ello es necesario destacar que el trabajo de la brigada ha sido motivo de reconocimiento e inspiración a nivel Interinstitucional, donde se ha tenido la oportunidad de compartir la experiencia dentro del congreso: "Reunión Interinstitucional del Sector Salud en Materia de Protección Civil Gestión del Riesgo", siendo la sede el Instituto Nacional de Rehabilitación, dando a conocer a nivel hospitalario que el INPer es la única institución con una brigada consolidada de Primeros Auxilios Psicológicos.

Por tanto, se pretende, como se está haciendo a lo largo de este tiempo, promover la importancia y el reconocimiento a la atención psicológica para brindar atención de manera integral a las personas, así como fomentar la prevención en el área de la salud mental desarrollando un trabajo de manera biopsicosocial a modo de prevención a las alteraciones psicológicas, patológicas y no patológicas. 


\section{Pronóstico}

\section{Descripción de la fase de seguimiento}

Se continuará con la promoción al trabajo psicoterapéutico, el cual se puede incorporar en diversos ámbitos, donde la mayor limitación radica en el desconocimiento y la estigmatización de la población, siendo éste un inconveniente para el aprovechamiento e inserción de los psicólogos.

De esta manera, con la ejemplificación de la experiencia institucional se busca difundir la importancia de la salud mental en México y la trascendencia a la actuación psicológica, pudiendo motivar a otras instituciones para la incorporación del psicólogo en diversos sectores laborales, y poner de igual manera el ejemplo a nivel internacional los trabajos de Martínez y Pery (2013) y Rotger (1999), quienes han incorporado el quehacer psicológico en las Fuerzas Armadas y al Cuerpo de Bomberos, para la actuación de desastres.

\section{Resultados en la fase de seguimiento}

Se concluye que el trabajo psicoterapéutico desarrollado en situaciones de desastre ha generado mayor entendimiento a la labor del psicólogo y la importancia hacia el cuidado de la salud mental, siendo un factor favorable para la erradicación de las limitaciones presentadas.

Por otra parte, la brigada de Primeros Auxilios Psicológicos, con el apoyo institucional, continuará atendiendo a la población para promover su estabilidad emocional, así como continuar con la sensibilización de las implicaciones emocionales en eventos críticos, considerando los aspectos contextuales y factores psíquicos de las personas, los cuales requieren atención especializada para la creación de una conciencia de prevención

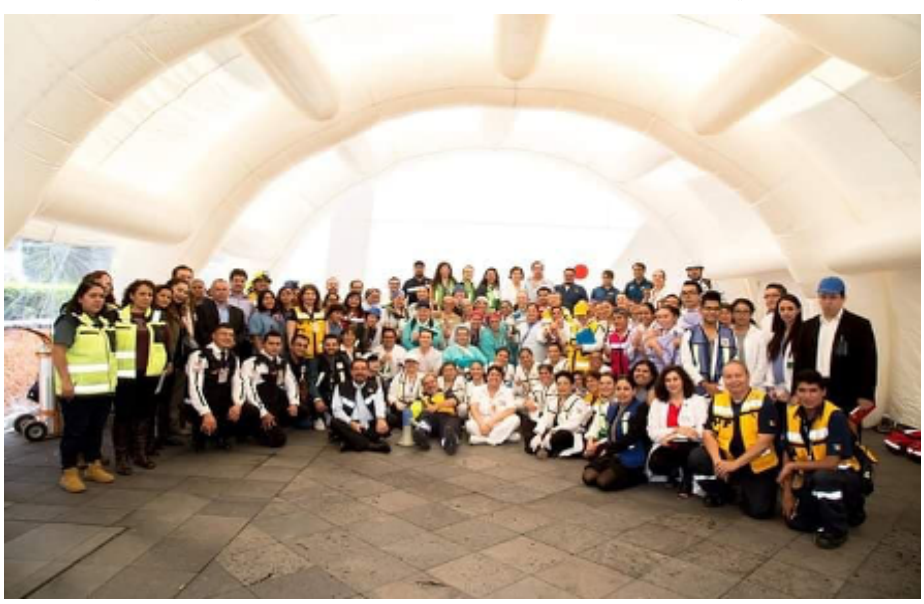

e importancia a la salud mental (figura 4).

Figura 4.

\section{Referencias}

Amenero, D., \& Huarcaya, J. (2019). Salud mental en situaciones de desastres. Horizonte Médico, 19(1), 73-80. Recuperado de $\quad$ http://www.scielo.org.pe/scielo.php?script=sci arttext\&pid=S1727-558X2019000100012

Bc Business Consultants Management S. C. (2018). Comando de incidentes. Material de aula. Instituto Nacional de Perinatología, Ciudad de México.

Centro Nacional de Prevención de Desastres (CENAPRED) (2018). Los desastres y sus efectos psicológicos. Material de aula online. Ciudad de México.

Centro Regulador de Emergencias Industriales Javier de la Madrid Torices (CREI) (2018). Sistema de Comando de Incidentes. Material de aula. Instituto Nacional de Perinatología, Ciudad de México.

Espíndola, J., Morales-Carmona, F., Díaz, E., Pimentel, D., Meza, P., Henales, C., Carreño, J., \& Ibarra, A. (2006). Malestar psicológico. Algunas de sus manifestaciones clínicas en la paciente gineco-obstétrica hospitalizada. Perinatología y Reproducción Humana, 20(4), 112,122. Recuperado de http://www.inper.mx/descargas/pdf

Flores, A. (2018). Generalidades de las emergencias y desastres hospitalarios. En S. Hernández (presidencia). Congreso llevado a cabo en el 2o. Curso de Intervenciones de Enfermería en Situaciones de Desastre, Ciudad de México. Instituto Nacional de Perinatología (INPer) (2017). Quienes Somos. Instituto Nacional De Perinatología. Recuperado de http://www.inper.mx/quienessomos/.

Martínez, J., \& Pery, L. (2013). Intervención en situaciones de crisis. Primeros auxilios psicológicos. Revista General de Marina, 265(3), 501-516. Recuperado de https://dialnet. unirioja.es/servlet/autor?codigo $=3335062$.

Morales-Carmona, F., \& Díaz-Franco, E. (2000). Algunas consideraciones sobre la psicología en las instituciones médicas. Perinatología y Reproducción Humana, 14(3), 176-182. Recuperado de https://www.medigraphic.com/ pdfs/inper/ip-2000/ip003g.pdf.

Organización Mundial de la Salud (OMS) (2020). Las emergencias y las personas de edad. Recuperado de https://www.who. int/ageing/projects/emergencies/es/.

Rabelo, J. (2010). Primeros auxilios psicológicos e intervención en crisis desde el trabajo social. Documentos de trabajo social. Revista de trabajo y acción social, 47, 121-133. Recuperado de https://dialnet.unirioja.es/servlet/ articulo?codigo $=3655753$.

Real Academia Española (RAE) (2020). Disquisición. En Diccionario de la lengua española, 22a. ed.). Recuperado de https://dle.rae.es/emergencia?m=form.

Rotger, D. (1999). La preparación psicológica del bombero y otros miembros de grupos de rescate. Su aceptación al estrés y los primeros auxilios psicológicos a las víctimas. Mapfre Seguridad, 74(2), 23-33. Recuperado de https:// www.fundacionmapfre.org/documentacion/publico/ i18n/consulta/registro.cmd?id=52397.

Segundo, S. (2018). Efectos de las emergencias y desastres en los servicios de salud. En S. Hernández (presidencia). Congreso llevado a cabo en el 2o. Curso de Intervenciones de Enfermería en Situaciones de Desastre, Ciudad de México.

Tamariz, E. (2018). Tipos de emergencia y desastre. En S. Hernández (presidencia). Congreso llevado a cabo en el 2o. Curso de Intervenciones de Enfermería en Situaciones de Desastre, Ciudad de México. 


\section{Meta-Análisis del Artículo}

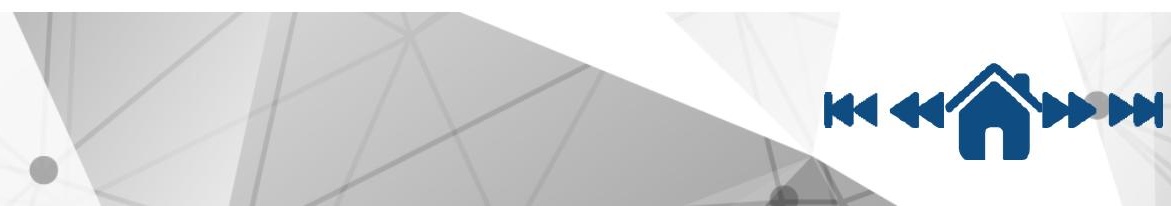




\section{Dimensión Cuantitativa}

\section{Perfil de Evaluación entre pares}
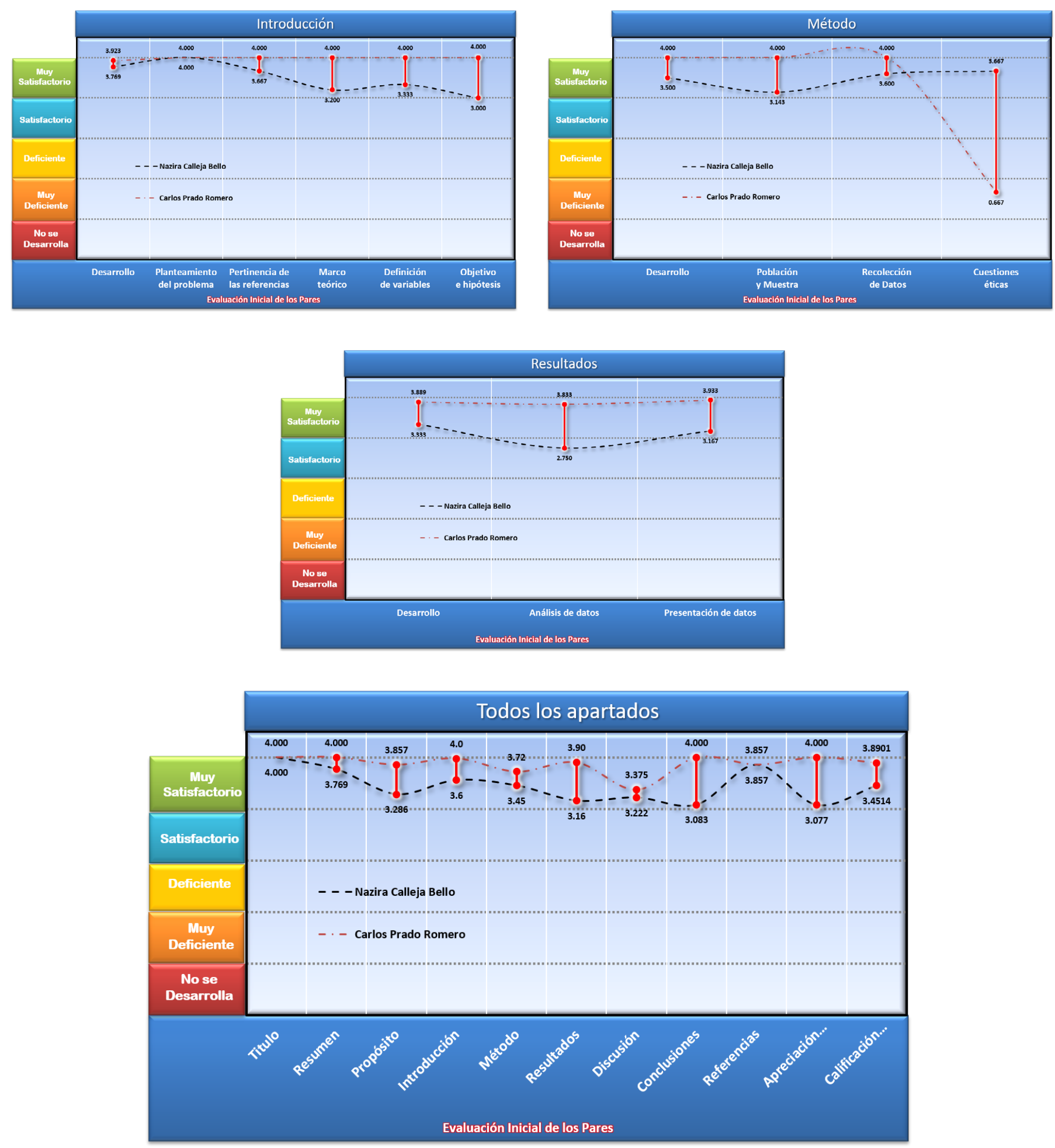


\section{Índice de Concordancia}

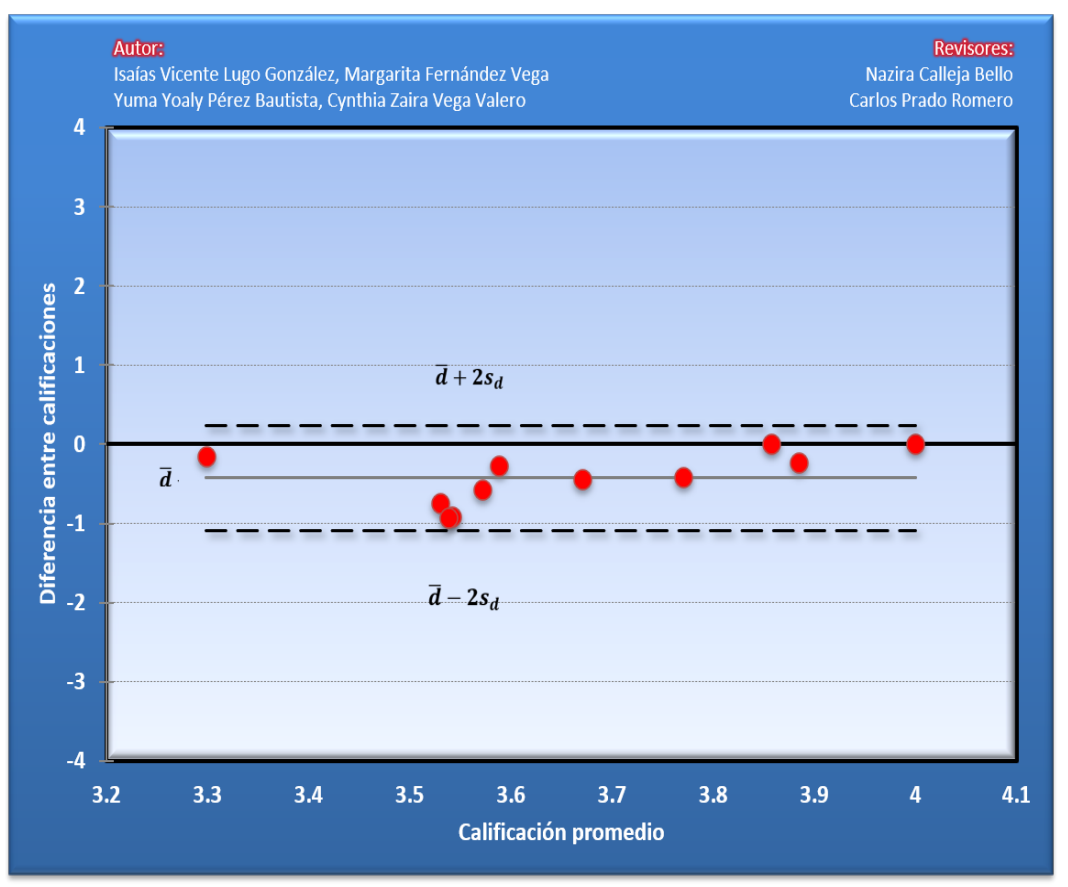

Índice de Acuerdo

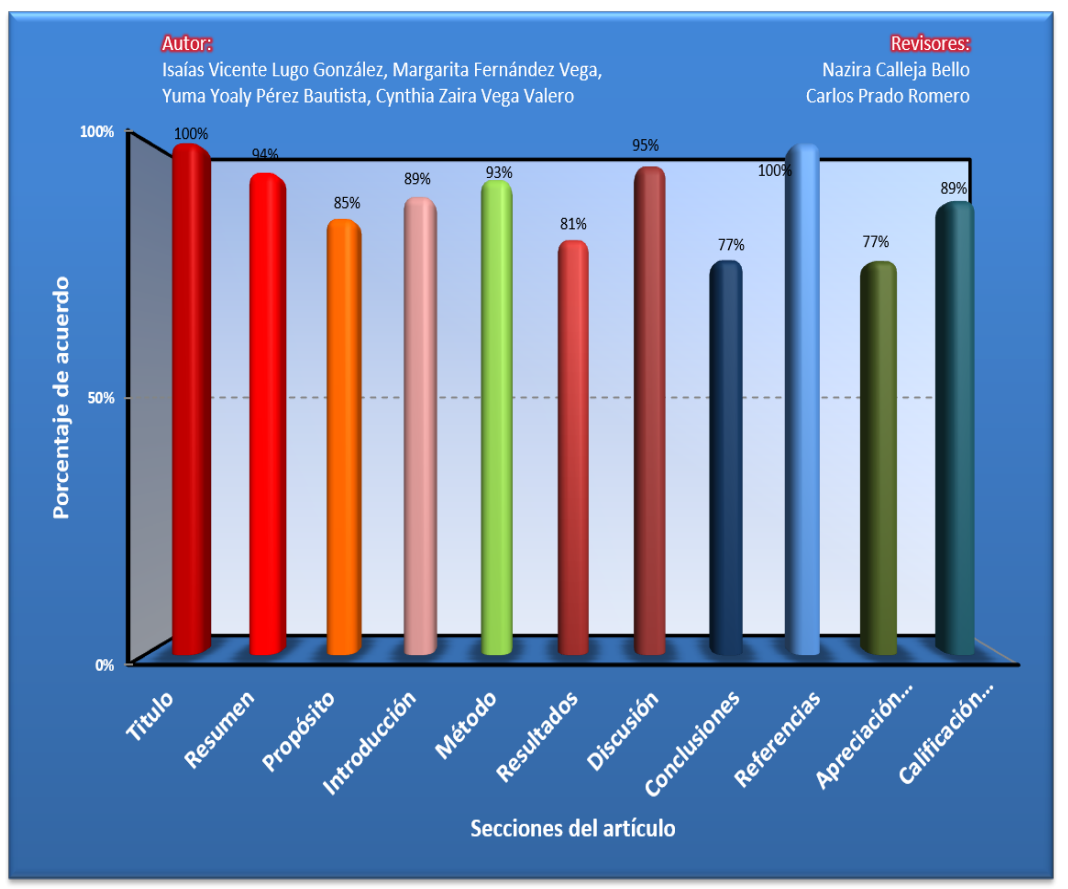




\begin{tabular}{|c|c|}
\hline Revisor 1 & Revisor 2 \\
\hline Patricia Balcázar Nava & Alicia Ivet Flores Elvira \\
\hline \multicolumn{2}{|c|}{ Título/Autoría } \\
\hline $\begin{array}{l}\text { No existen observaciones referentes al título en cuanto } \\
\text { a constructos, contenido, extensión ni uso de términos. } \\
\text { Es adecuado }\end{array}$ & No hay observaciones acerca del título. \\
\hline \multicolumn{2}{|c|}{ Resumen } \\
\hline $\begin{array}{l}\text { No existen observaciones respecto del resumen y su equi- } \\
\text { valente en inglés en cuanto a forma, contenido, redacción. }\end{array}$ & $\begin{array}{l}\text { El resumen no se organiza en párrafos, sobre resultados } \\
\text { es muy breve la descripción. Consta de } 190 \text { palabras, } \\
\text { presenta } 4 \text { palabras clave (se recomienda agregar crisis). }\end{array}$ \\
\hline \multicolumn{2}{|c|}{ Próposito del Estudio } \\
\hline $\begin{array}{l}\text { No existen comentarios al respecto del objetivo del es- } \\
\text { tudio, siendo un estudio de caso, se respeta la meto- } \\
\text { dología y la esencia de lo que debe presentarse en un } \\
\text { reporte de esta naturaleza }\end{array}$ & $\begin{array}{l}\text { Se establece como eje de la intervención la brigada de } \\
\text { primeros auxilios psicológicos, sin embargo al presen- } \\
\text { tar resultados se habla del simulacro en general, sin pre- } \\
\text { sentar datos específicos sobre las dos intervenciones en } \\
\text { crisis que se mencionan se realizaron. Hay mención de } \\
\text { las acciones de intervención en crisis aunque no se pre- } \\
\text { senta con mayor detalle cómo valoraron la eficiencia de } \\
\text { las medidas o bien de la ejecución de los brigadistas que } \\
\text { participaron en este ejercicio (dado que hacen énfasis en } \\
\text { analizar la capacitación que reciben los brigadistas). }\end{array}$ \\
\hline \multicolumn{2}{|c|}{ Introducción } \\
\hline $\begin{array}{l}\text { Sería deseable que se identifique la problemática que in- } \\
\text { dican al inicio, tratando de indicar si existen modelos en } \\
\text { otros hospitales en México o en el mundo, que esté traba- } \\
\text { jando con el modelo que presenta, además de indicar en } \\
\text { el tiempo cuáles han sido los avances de programas de este } \\
\text { tipo, para que el lector tenga más claridad sobre el tema y } \\
\text { se vea la relevancia del tipo de caso que están reportando. }\end{array}$ & $\begin{array}{l}\text { La introducción se organiza más como un reporte de } \\
\text { experiencia institucional, sin hacer explícitos los ele- } \\
\text { mentos que destacarían la vertiente de investigación } \\
\text { (hipótesis, objetivos, variables). }\end{array}$ \\
\hline
\end{tabular}

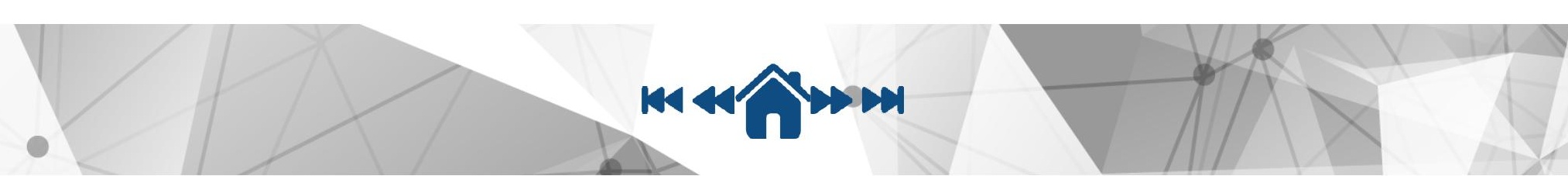




\begin{tabular}{|c|c|}
\hline Revisor 1 & Revisor 2 \\
\hline \multicolumn{2}{|c|}{ Método } \\
\hline $\begin{array}{l}\text { Se vislumbra congruencia en las consideraciones éti- } \\
\text { cas, desarrollo del estudio de caso, recolección de datos } \\
\text { y la descripción de la población. }\end{array}$ & $\begin{array}{l}\text { El documento de manera general lleva un formato de } \\
\text { reporte de experiencia sintetizado, no contempla ele- } \\
\text { mentos que destaquen la dimensión de investigación. } \\
\text { Parecía que el eje del trabajo sería la intervención de } \\
\text { índole psicológico, pero se presenta más información } \\
\text { general sobre el simulacro de sismo. }\end{array}$ \\
\hline \multicolumn{2}{|c|}{ Resultados } \\
\hline $\begin{array}{l}\text { Existe congruencia entre el objetivo, los resultados, la pre- } \\
\text { sentación de los mismos y el tipo de análisis efectuado }\end{array}$ & $\begin{array}{l}\text { Se describe de manera general la intervención realizada } \\
\text { en el simulacro, pero no se detalla lo que se realizó en } \\
\text { concreto sobre primeros auxilios psicológicos. }\end{array}$ \\
\hline \multicolumn{2}{|c|}{ Discusión } \\
\hline $\begin{array}{l}\text { Sería deseable incluir resultados de otros casos para } \\
\text { tratar de comparar y de vislumbrar la importancia de } \\
\text { los hallazgos }\end{array}$ & $\begin{array}{l}\text { Al cierre del texto se presentan interpretaciones de la } \\
\text { experiencia sin embargo no se sustentan en datos de la } \\
\text { intervención realizada o de los efectos que indican que } \\
\text { se generaron en consecuencia (avance e impacto en la } \\
\text { percepción a la prevención de riesgos y la importancia } \\
\text { de la Salud Mental). }\end{array}$ \\
\hline \multicolumn{2}{|c|}{ Conclusiones } \\
\hline $\begin{array}{l}\text { Se propone se incluyan datos de otras investigaciones } \\
\text { similares para abonar más a la importancia del estudio } \\
\text { de caso reportado }\end{array}$ & $\begin{array}{l}\text { Se mencionan propuestas a futuro relacionadas con la inter- } \\
\text { vención directa sin que se asocien a fines de investigación. }\end{array}$ \\
\hline \multicolumn{2}{|c|}{ Referencias } \\
\hline $\begin{array}{l}\text { Si se considera el estilo de la APA, existen algunos fal- } \\
\text { tantes tanto en la forma de colocar las referencias (en } \\
\text { orden alfabético y no en orden de aparición de las citas), } \\
\text { además de que existen algunos detalles relacionados con } \\
\text { el estilo de la referencia y el estilo de las citas que hay } \\
\text { que cuidar. También se sugiere se incremente el número } \\
\text { de referencias relacionadas con el objetivo de estudio en } \\
\text { otros casos de corte nacional e internacional }\end{array}$ & $\begin{array}{l}\text { Hay una referencia donde probablemente sea necesario } \\
\text { cambiar la mención del autor. No se sigue orden alfabético. }\end{array}$ \\
\hline
\end{tabular}




\section{Historia del Proceso}

\section{EDITORIAL}

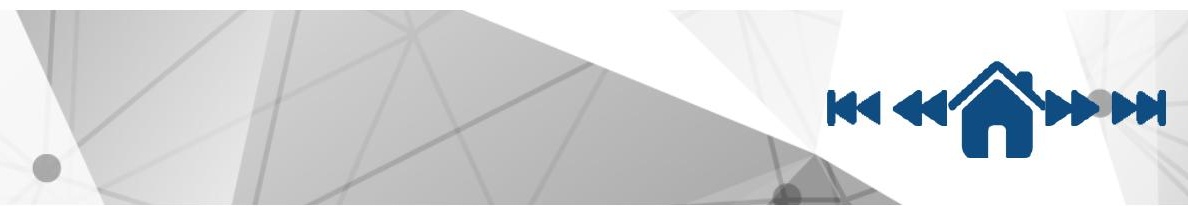

